\title{
NEAR at Mathilde and Eros: An Update
}

\author{
Andrew Cheng \\ Johns Hopkins Applied Physics Laboratory, MD, USA
}

At the slowly rotating C-asteroid Mathilde, the Near Earth Asteroid Rendezvous (NEAR) mission imaged only one face of the body but still constrained the density to only $1.3 \mathrm{~g} / \mathrm{cm}^{3}$, indicating high porosity. This porosity and the approximate saturation of the surface with giant craters led to suggestions that Mathilde must be a rubble pile. At S-type Eros, NEAR measured elemental abundances and spatially resolved reflectance spectra that appear most consistent with an ordinary chondrite composition, although the Eros composition could not be identified with any known meteorite type. Imaging and altimetry data were interpreted as showing Eros to be a globally consolidated, albeit heavily fractured, collisional shard. A spectacular feature, ponding of regolith on Eros, was discovered. Continuing analyses of NEAR data suggest that Mathilde is not strengthless, and that if it is a rubble pile, large coherent components are present. Both composition and internal structure of Eros pose unresolved issues. Although almost uniform internally, Eros cannot be perfectly so. The density of $2.67 \mathrm{~g} / \mathrm{cm}^{3}$ is significantly less than that of ordinary chondrites and suggests significant porosity, but this may not be explained by macroscopic fracturing if these spaces can be filled with particulates. 